\title{
Chronic rhinosinusitis with nasal polyposis: the role of personalized and integrated medicine
}

\author{
Giorgio Ciprandi ${ }^{1}$, Matteo Gelardi ${ }^{2}$ \\ ${ }^{1}$ Allergy Clinic, Casa di Cura Villa Montallegro, Genoa; ${ }^{2}$ Department of Otolaryngology, University of Foggia, Italy
}

\begin{abstract}
Chronic rhinosinusitis with nasal polyposis (CRSwNP) is a common disorder. From a clinical and immunopathological point of view, different phenotypes and endotypes have been identified. As asthma is frequent comorbidity, biological agents for treating CRSwNP associated with asthma may be an attractive strategy. Biological agents have several mechanisms, such as antagonizing IgE, interleukin (IL) 4, IL-5, and IL-13. However, a workup is mandatory, mainly concerning pheno-endotyping. In this regard, clinical cytological grading $(\mathrm{CCG})$ has been proposed as a useful tool to manage patients with CRSwNP as it allows us to define clinical and immunopathological phenotypes able to identify the ideal candidate for biologics. In particular, the mixed cellular pattern, such as eosinophils and mast cells, could be sensitive to antiIL-4 agents. There is still a need for well-established indications, criteria of responsiveness, duration, and safety. Moreover, personalized medicine could be opportunely integrated and/or alternated with intranasal corticosteroids to prevent relevant adverse events.
\end{abstract}

Correspondence: Giorgio Ciprandi, Allergy Clinic, Casa di Cura Villa Montallegro, Via Monte Zovetto 27, 16145 Genova, Italy.

E-mail: gio.cip@libero.it

Ethics approval and consent to participate: Not applicable.

Availability of data and material: Not applicable.

Conflict of interest: The authors declare that they have no competing interests, and all authors confirm accuracy.

Contributions: GC, designed the paper and wrote the manuscript; MG, discussed it. Both authors approved the manuscript.

Key words: chronic rhinosinusitis, nasal polyposis, clinical-cytological-grading, biological agents, corticosteroids.

Received for publication: 14 January 2021

Accepted for publication: 24 February 2021.

${ }^{\circ}$ Copyright: the Author(s), 2021

Licensee PAGEPress, Italy

Monaldi Archives for Chest Disease 2021; 91:1769

doi: 10.4081/monaldi.2021.1769

This article is distributed under the terms of the Creative Commons Attribution Noncommercial License (by-nc 4.0) which permits any noncommercial use, distribution, and reproduction in any medium, provided the original author(s) and source are credited.

\section{Introduction}

Chronic rhinosinusitis (CRS) represents a common disease as it affects about $10-12 \%$ of the European population [1]. CRS consists of two main phenotypes based on endoscopy and computed tomography (CT) findings: CRS with nasal polyposis (CRSwNP) and CRS without nasal polyposis (CRSsNP) [2]. CRSwNP is defined by the presence of nasal polyps and signs and symptoms lasting longer than $8-12$ weeks $[3,4]$. The CRSwNP overall prevalence is approximately estimated to be $2 \%$ to $4 \%$ of the general population. From a pathophysiological point of view, nasal polyps are benign edematous masses in the nasal cavities, paranasal cavities, or both. Their occurrence depends on an exaggerated inflammatory reaction. Type 2 inflammation usually subtends the polyps' generation [2,3]. Type 2 immune response is characterized by a $\mathrm{T}$ helper 2 polarization, sensitization to allergens, and eosinophilic infiltrate. As nasal polyps occupy space into nasal cavities, they can cause nasal obstruction; moreover, the surrounding inflammation leads to rhinorrhea, postnasal drip, hypo-, or anosmia [5].

Treatment options for CRSwNP consist of local or systemic corticosteroids as the first-line choice. If ineffective, there is a need for functional endoscopic sinus surgery. Notably, patients with CRSwNP and comorbid asthma have insufficient therapeutic responses and a high recurrence rate, so the disease is more challenging to treat. Both CRSwNP and asthma share a severe impairment of quality of life (QoL) and cause a substantial financial burden for society [3]. On the other hand, recent studies provided evidence about the phenotypes and the endotypes of chronic respiratory disorders, mainly concerning inflammation and the type of immune response [6-9]. As a result, more targeted, effective, and efficient Precision Medicine (PM) has been developed. PM refers to the "ability to identify subpopulations that differ in their susceptibility to a particular disease, in the biology or prognosis of those diseases they may develop, or in their response to a specific treatment" [10]. Consequently, PM allows us to stratify patients into subgroups and tailor treatment based on their peculiar phenoendotypes [11]. This approach has been defined as Personalized Medicine [12].

\section{Practical aspects}

CRSwNP is frequently associated with asthma; it was reported that among patients with CRSwNP, approximately $30 \%$ had asthma, and $15 \%$ had aspirin intolerance [13]. On the other hand, asthma is a chronic inflammatory disease of the lower airways characterized by bronchial inflammation, airway hyperresponsiveness, 
and usually reversible airflow obstruction, leading to recurrent wheezing episodes, breathlessness, chest tightness, and coughing [14]. Asthma has a high prevalence, ranging from $5-10 \%$ of the general population [15]. Asthma is frequently associated with many comorbidities, including upper airways, rhinitis, and rhinosinusitis $[16,17]$. In particular, the CRSwNP-asthma phenotype, characterized by the association of CRSwNP with asthma, usually displays particularly severe progress [18], and the treatment costs are significant [19].

Biologics significantly changed the possibility of treating type 2-mediated inflammatory diseases. The first medication was omalizumab, initially approved for the therapy of moderate-tosevere perennial allergic asthma [20]. As CRSwNP share similar pathogenic mechanisms with asthma, mainly concerning type 2 inflammation, the possible indication for nasal polyps was further required $[21,22]$. Many trials have been conducted using biologics for treating CRSwNP in these last years [23-28]. The European Forum for Research and Education in Allergy and Airway Diseases EUFOREA has recently provided a consensus on this issue [29].

The rationale for using biologics in CRSwNP is based on the existence of clinical-morphological characteristics and unbalanced immune responses that define specific pheno-endotypes [30]. CRSwNP may be classified based on the type of immunologic response. Type 1 immune response is sustained by a Th1 polarization associated with neutrophilic infiltration and IFN-y overproduction; this endotype is most common in Asia [31]. Type 2 inflammation includes interleukin 4 (IL-4), IL-5, and IL-13 over-expression and nasal eosinophilia; comorbidity with asthma and allergic diseases is frequent [32]. Type 3 immune response is characterized by increased IL-17 cytokine family release and frequent exacerbations [33]. CRSwNP is a pleiomorphic disorder; for example, the type 1 NP may include other phenotypes, such as cilia motility defects, cystic fibrosis, and infectious sinus diseases [34]. These last conditions are frequent in young adults [35]. Moreover, a mixed type 1 and type 2 endotype has also been reported and imbrications with type 3 endotype [36]. This endotype pleiomorphism might depend on the plasticity of type 2 innate lymphoid cells [37]. These cells amplify type 2 inflammation independently of allergy [39]. A typical example is the nonallergic rhinitis with eosinophils (NARES), characterized by nasal eosinophilia without sensitization [39].

Clustering patients initially considered symptoms and quality of life aspects [40,41]. Successively, immunological parameters were also measured in patients suffering from CRSwNP, including IL- 5, eosinophilic cationic protein (ECP), S. aureus enterotoxin (SE-IgE), and albumin to define endotypes [42].

\section{Pragmatic approach}

Usually, a clinical approach is used to define algorithms, including an assessment of the visual analog scale (VAS) and the sino-nasal outcome test (SNOT-22) score [40,41]. Biomarkers were also investigated to improve predictive criteria [28]. Initially, tissue eosinophilia and IgE (classical type 2 biomarkers) were used to predict corticosteroid sensitivity [42]. Other biomarkers were evaluated, including 24-h urinary LTC4 $[43,44]$.

However, no reliable biomarker could classify type 1 endotype and anti-type 3 targeted biologics that were ineffective in the asthma model [45-48].

\section{Trials with biological agents}

Some trials provided evidence about the effectiveness of biologics in the treatment of CRSwNP. The first experience was conducted in patients with severe asthma, also observing unexpected improvement of NP. Consequently, different molecules were tested on this topic.

Omalizumab, an anti-IgE monoclonal, was the first experienced biologic in the treatment of patients with CRSwNP. A series of convincing proofs have been documented both by randomized controlled trials $[21,49,50]$ and real-life studies [51]. Interestingly, omalizumab was effective in treating NP also in non-allergic patients [21,52]. This outcome could open the possibility to explore new indications for this biological agent.

Mepolizumab is an anti-IL-5 monoclonal antibody that reduces peripheral and bronchial eosinophils in asthmatic patients [53]. Mepolizumab has been investigated successfully also in patients with CRSwNP [54,55]. In particular, it has been recently reported that mepolizumab could reduce the need for sinus surgery [56].

Reslizumab is an anti-IL-5 monoclonal antibody that binds to IL-5, preventing it from binding the IL-5 receptor's subunit. The effects of reslizumab on patients with asthma and self-reported nasal polyposis [57].

Benralizumab is an anti-IL-5 monoclonal antibody that binds to the IL-5 receptor's a-chain, initiating a direct, rapid depletion of eosinophils through enhancing the antibody-dependent cell-mediated cytotoxic pathway via the NK cells [58]. A case report of severe asthma with eosinophilic CRS has been successfully treated with benralizumab [59].

Dupilumab is an anti-IL-4 monoclonal antibody that targets the alpha chain of IL-4Ra, a common receptor for IL-4 and IL-13. These two cytokines play a prominent role in the Th2 pathway and nasal polyp formation [60]. In a randomized, double-blind, placebo-controlled phase 2 study of dupilumab in patients with CRSwNP with and without asthma, the dupilumab group experienced significant improvement in endoscopic, radiographic, and QoL endpoints relative to placebo [61]. These clinical changes were accompanied by a statistically significant reduction in circulating concentrations of the type 2 biomarkers, such as total serum $\operatorname{IgE}$ and eotaxin-3. More recently, it has been reported that dupilumab reduced biomarkers of type 2 inflammation, including eotaxin, eotaxin 3, eosinophilic cationic protein, in polyps of patients with CRSwNP [62]. In June 2019, the U.S. Food and Drug Administration (FDA) approved dupilumab to treat adults with inadequately controlled CRSwNP.

The efficacy and safety of dupilumab were established in two studies (one lasting 24 weeks and the other 52 weeks: SINUS-24 and SINUS-52, respectively), including 724 adult patients with severe CRSwNP who remained symptomatic despite taking INCS [63]. Patients who received add-on dupilumab had a statistically significant reduction in their nasal polyp size, CT scores, and nasal congestion compared to the placebo group. The Dupilumab group also improved the ability to smell and required less nasal polyp surgery and oral steroids [63]. Improvement of FEV1 was also reported in treated patients with concomitant asthma, highlighting the finding that dupilumab may simultaneously address multiple comorbid manifestations of type 2 inflammatory diseases occurring in both the upper and lower airways. During the study period, dupilumab was generally well tolerated and had an acceptable safety, with nasopharyngitis, epistaxis, and injection-site reactions reported as the most common adverse events in the treated group 
[63]. As a result, dupilumab is the only biologic approved for the treatment of CRSwNP at present.

\section{Biomarkers in clinical practice}

These reported outcomes were encouraging and could pave the avenue to a new promising approach in patients with CRSwNP even though a need for a precise classification of the patients is still mandatory [64]. CRSwNP is a multifaceted disease frequently characterized by multiple phenotypes- endotypes. Patients with CRSwNP may belong to type 1, type 2, or type 3 endotype but could display mixed phenotypes and multiple endotypes. In this regard, four biomarkers could differentiate type 2 from non-type 2 inflammation: serum specific IgE, peripheral eosinophils, nasal cytology, and fractional exhaled nitric oxide (FeNO), which are readily available in daily clinical activity [65]. The demonstration of allergenspecific IgE production diagnoses allergy. IgE production, such as sensitization, is the conditio sine qua non to define allergy [66]. Peripheral blood eosinophils correlated well with nasal eosinophils; thus, peripheral eosinophils could be reasonably considered a biomarker for suspecting type 2 inflammation also at the nasal level [67]. Nasal cytology may document eosinophilic infiltrate [68]. FeNO identifies type 2 bronchial inflammation associated with eosinophil activation [69]. NO can also be measured at the nasal level [70]. Type 2 immune response could be stratified into two subgroups: the allergic endotype and the non-allergic endotype.

\section{Personalized and integrated treatments}

As previously discussed, the current challenge for the doctor managing subjects with CRSwNP is choosing the more appropriate therapy for the single patient, hopefully, according to the Precision Medicine and the Personalized Medicine approach. In this regard, a multidisciplinary board of the EUFOREA suggested biologics' positioning in this topic [29]. First, a careful selection of patients was recommended: five prescriptive criteria were identified: i) evidence of type 2 inflammation, ii) need of systemic corticosteroids in the past two years, iii) significant QoL impairment, iv) significant hyposmia, and v) asthma comorbidity. In this regard, we would underline the frequent CRSwNP comorbidity in patients with asthma. A recent study showed that $35 \%$ of asthmatics had concomitant CRS [71]. This high prevalence suggests the clinical recommendation of carefully evaluating patients with asthma to verify possible CRSwNP comorbidity.

Contraindications could be CRSsNP diagnosis, non-type 2 inflammation, cystic fibrosis, unilateral polyps, mucoceles, immunodeficiency, and factors associated with poor compliance. Another relevant point was the recognition of criteria for defining response to biological therapy after one year: reduced nasal polyp size, reduced need for systemic corticosteroids, improved QoL, improved olfaction, and reduce the impact of comorbidities [29]. Moreover, as carefully pointed out by the Consensus, a multidisciplinary integrated care pathway should be performed in clinical practice. A thorough evaluation of both upper and lower airways should be done at every visit, monitoring symptoms, available biomarkers, airway function, and control medications use.

On the other hand, it has to be underlined that the enthusiastic interest obtained by biological agents should not obfuscate the relevant importance of the so-called small molecule drugs (SMD), as recently highlighted by an EAACI Taskforce on Immuno-pharmacology [72]

SMD is an umbrella definition that includes several medications belonging to different classes, such as topical and systemic corticosteroids, antagonists of leukotrienes, $\beta 2$-agonists, antimuscarinic agents, mast cell stabilizers, and other active compounds [72]. In this regard, intranasal corticosteroids represent the firstline choice in the management of CRSwNP $[3,4]$. Intranasal corticosteroids could be opportunely integrated with biologics in these patients.

\section{CRSwNP grading}

In precision medicine, and as a consequence of personalized medicine, it is crucial to identify the patients who will be candidates for biological therapy. Furthermore, it is also essential to monitor these patients in an objective and re-educable way. In this context, the possibility of evaluating patients with CRSwNP using clinical-cytological grading has existed for several years $[73,74]$. This tool allows us to obtain useful clinical information that can be collected over time. The GCC is also associated with the sense of smell impairment [75]. In particular, CCG evaluates the presence of comorbidity, including asthma, allergy, and aspirin intolerance, and the pattern of the cellular infiltrate, including eosinophils, neutrophils, and mast cells. CCG global score is classified as lowgrade (score 1-3), medium-grade (4-6), and high-grade ( $\geq 7$ ).

A useful CCG implementation in clinical practice has been recently investigated in a retrospective cross-sectional real-world study [76]. The study recruited 791 outpatients with CRSwNP (424 males, mean age 48.8 years). In the total population, asthma was a common comorbidity $(30.8 \%)$ as well as aspirin intolerance $(24.8 \%)$, and allergy (50.8\%); $210(26.5 \%)$ outpatients had low-grade, 366 (46.3\%) medium-grade, and $215(27.2 \%)$ high-grade CCG. As regards cytological phenotypes, 87 (11\%) had neutrophilic type, 371 (46.3\%) eosinophilic, 112 (14.2\%) mast cell, and 221 (27.9\%) mixed. High-grade CCG was significantly associated with more frequent asthma, aspirin intolerance, allergy, recurrent surgery, and mixed cytological phenotype. Low-grade CCG was characterized by fewer comorbidities and surgery for polyps and neutrophilic phenotype.

Consequently, CCG may be considered a useful tool in managing outpatients with CRSwNP as it allows an optimal identification of the best-tailored therapy, as recently pointed out [77]. Another study is still ongoing and aimed at correlating CCG with SNOT-22, a standard questionnaire used in CRSwNP patients [78]. The preliminary results confirmed the clinical relevance of $\mathrm{CCG}$ as its outcomes are well related to SNOT-22 findings. Therefore, CCG assessment could help identify the candidate for biologics, mainly concerning patients with a mixed cellular pattern, such as eosinophils and mast cells. Namely, these cells' massive presence corresponds to a type 2 high phenotype, which is highly sensitive to anti-IL-4 agents, including dupilumab [79].

\section{Conclusions}

Biological agents are a promising therapy for CRSwNP that could be adequately addressed to select patients after a careful workup, mainly considering the pathogenic mechanisms and comorbidity. The pathways and potential targets should be recognized, as summarized in Figure 1 [80]. In particular, patients with 


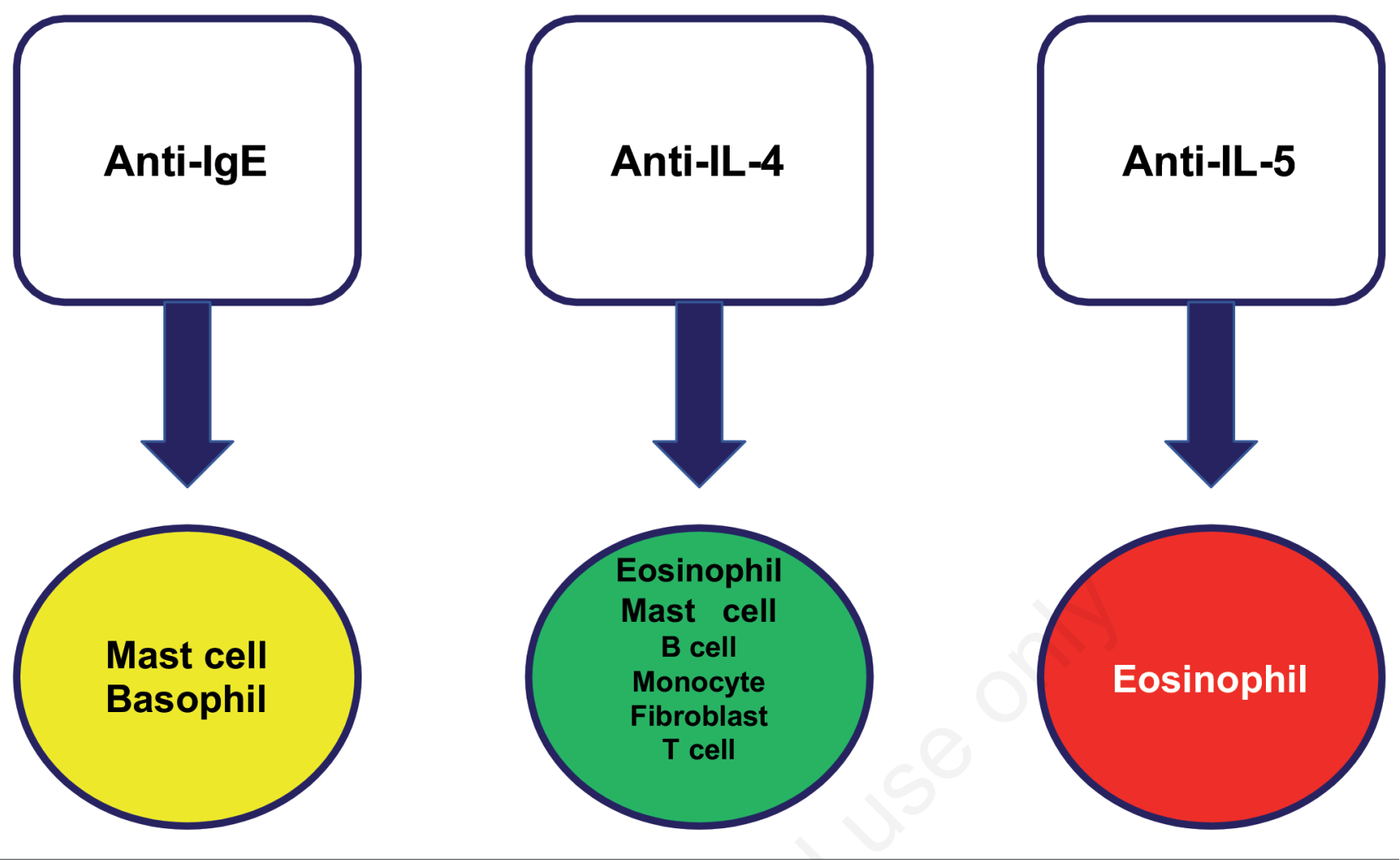

Figure 1. Schematic representation of the main mechanisms and targets of biologics in CRSwNP.

olfaction dysfunction could achieve a particular improvement [81]. Moreover, biologics for CRSwNP could be an attractive therapeutic strategy in patients with severe type-2 inflammation [82]. However, conventional anti-inflammatory therapy should be prescribed as effective, safe, and cheap. Of course, biologics and intranasal corticosteroids could be integrated between them. Therefore, personalized and integrated therapies could be favorably prescribed in patients with CRSwNP.

\section{References}

1. Hassan D, Fokkens WJ, Bachert C, Newson RB, Bislimovska J, Bockelbrink A, et al. Chronic rhinosinusitis in Europe - an underestimated disease. A GA(2)LEN study. Allergy 2011;66: 1216-23.

2. Bachert C, Zhang L, Gevaert P. Current and future treatment options for adult chronic rhinosinusitis: focus on nasal polyposis. J Allergy Clin Immunol 2015;136:1431-40.

3. Fokkens WJ, Lund VJ, Mullol J, et al. EPOS 2012: European position paper on rhinosinusitis and nasal polyps 2012. A summary for otorhinolaryngologists. Rhinology 2012;50:1-12.

4. Orlandi RR, Kingdom TT, Hwang PH, et al. International Consensus Statement on Allergy and Rhinology: Rhinosinusitis. Int Forum Allergy Rhinol 2016;6:S22-209.

5. Lund VJ, Mackay IS. Staging in rhinosinusitis. Rhinology 1993;31:183-4.

6. Bachert C, Gevaert P, Holtappels G, et al. Total and specific
IgE in nasal polyps is related to local eosinophilic inflammation. J Allergy Clin Immunol 2001;107:607-14.

7. Mygind N, Dahl R, Bachert C. Nasal polyposis, eosinophil dominated inflammation, and allergy. Thorax 2000;55:S79-83

8. Gevaert P, Bachert C, Holtappels G, et al. Enhanced soluble interleukin-5 receptor alpha expression in nasal polyposis. Allergy 2003;58:371-9.

9. Patou J, Holtappels G, Affleck K, et al. Enhanced release of IgE-dependent early phase mediators from nasal polyp tissue. J Inflamm (Lond) 2009;6:11.

10. Timmerman L. What's in a name? A lot, when it comes to 'precision medicine'. Xconomy [Internet]. 2013. Available from: https://xconomy.com/national/2013/02/04/whats-in-a-name-alot-when-it-comes-to-precision-medicine/

11. Kaur R, Chupp G. Phenotypes and endotypes of adult asthma: moving toward precision medicine. J Allergy Clin Immunol 2019;144:1-12.

12. Guilleminault L, Ouksel H, Belleguic C, et al. Personalised medicine in asthma: from curative to preventive medicine. Eur Respir Rev 2017;26:160010.

13. Bachert C, Vignola AM, Gevaert P, et al. Allergic rhinitis, rhinosinusitis, and asthma: one airway disease. Immunol Allergy Clin North Am 2004;24:19-43.

14. Global Initiative for Asthma. GINA guidelines. Global Strategy for Asthma Management and Prevention. 2019. Accessed on: September 2019. Available from: htpp://www. ginasthma.org/

15. GBD 2015 Chronic Respiratory Disease Collaborators. Global, regional, and national deaths, prevalence, disabilityadjusted life years, and years lived with disability for chronic 
obstructive pulmonary disease and asthma, 1990-2015: a systematic analysis for the Global Burden of Disease Study 2015. Lancet Respir Med 2017;5:691-706.

16. Ciprandi G, Cirillo I. The lower airway pathology of rhinitis. Update Review. J Allergy Clin Immunol 2006;118:1105-9.

17. Tay TR, Hew M. Comorbid "treatable traits" in difficult asthma. Current evidence and clinical evaluation. Allergy 2018;73:1369-82.

18. Lin DC, Chandra RK, Tan BK, et al. Association between severity of asthma and degree of chronic rhinosinusitis. Am J Rhinol Allergy 2011;25:205-8.

19. Chung KF, Wenzel SE, Brozek JL, et al. International ERS/ATS guidelines on definition, evaluation, and treatment of severe asthma. Eur Respir J 2014;43:343- 73.

20. Casale TB, Israel E. Biologics, clinical context, and the asthmas. J Allergy Clin Immunol Pract 2019;7:1437-9.

21. Gurrola J II, Borish L. Chronic rhinosinusitis: endotypes, biomarkers, and treatment response. J Allergy Clin Immunol 2017; 140:499-508.

22. Tsetsos N, Goudakos JK, Daskalakis D, et al. Monoclonal antibodies for the treatment of chronic rhinosinusitis with nasal polyposis: a systematic review. Rhinology 2018;56:11-21.

23. Gevaert P, Calus L, Van Zele T, et al. omalizumab is effective in allergic and non-allergic patients with nasal polyps and asthma. J Allergy Clin Immunol 2013;131:110-6.

24. Bachert C, Manning L, Naclerio RM, et al. Effect of subcutaneous dupilumab on nasal polyp burden in patients with chronic sinusitis and nasal polyposis: a randomized clinical trial. JAMA 2016;315:469-79.

25. Bachert C, Sousa AR, Lund VJ, et al. Reduced need for surgery in severe nasal polyposis with mepolizumab: a randomized trial. J Allergy Clin Immunol 2017;140:1024-1031.e14.

26. Bidder T, Sahota J, Rennie C, et al. Omalizumab treats chronic rhinosinusitis with nasal polyps and asthma together-a real-life study. Rhinology 2018;56:42-5.

27. Fokkens WJ, Bachert C, Bernal-Sprekelsen M, et al. Rhinology future debates, a EUFOREA report. Rhinology 2017;55: 298-304

28. Hellings PW, Akdis CA, Bachert C, et al. EUFOREA rhinology research forum 2016: report of the brainstorming sessions on needs and priorities in rhinitis and rhinosinusitis. Rhinology 2017;55:202-10.

29. Fokkens WJ, Lund V, Bachert C, et al. EUFOREA consensus on biologics for CRSwNP with or without asthma. Allergy 2019;74:2312-9.

30. Vlastos I, Gkouskou K, Doulaptsi M, et al. Precision medicine in rhinosinusitis. Curr Allergy Asthma Rep 2019;19:12.

31. Dennis SK, Lam K, Luong A. A review of classification schemes for chronic rhinosinusitis with nasal polyposis endotypes. Laryngoscope Investig Otolaryngol. 2016;1:130-4.

32. Liu XJ, Liao B, Yu QH, et al. IL-37-Mex3B-TLR3 axis in epithelial cells in eosinophilic chronic rhinosinusitis with nasal polyps. J Allergy Clin Immunol 2020;145:160-72.

33. Ricciardolo F, Sorbello V, Folino A, et al. Identification of IL$17 \mathrm{~F} /$ frequent exacerbator endotype in asthma. J Allergy Clin Immunol 2017; 140:395-406.

34. Bachert C, Akdis CA. Phenotypes and emerging endotypes of chronic rhinosinusitis. J Allergy Clin Immunol Pract 2016;4: 621-8.

35. Sommer DD, Rotenberg BW, Sowerby LJ, et al. A novel treatment adjunct for aspirin-exacerbated respiratory disease: the low-salicylate diet: a multicenter randomized control crossover trial. Int Forum Allergy Rhinol 2016;6:385-91.
36. De Greve G, Hellings PW, Fokkens WJ, Pugin B. Endotypedriven treatment in chronic upper airway diseases. Clin Transl Allergy 2017;7:22.

37. Tomassen P, Vandeplas G, van Zele T, et al. Inflammatory endotypes of chronic rhinosinusitis based on cluster analysis of biomarkers. J Allergy Clin Immunol 2016;137:1449-1456.e4.

38. White AA, Doherty TA. Role of group 2 innate lymphocytes in aspirin-exacerbated respiratory disease pathogenesis. Am J Rhinol Allergy 2018;32:7-11.

39. de Campos L, Galvão CES, Mairena EC, et al. Increased gene expression of inflammatory markers in nasal turbinate of patients with persistent allergic rhinitis and chronic obstruction. Eur Arch Otorhinolaryngol 2019;276:3247-9.

40. Soler ZM, Hyer JM, Ramakrishnan V, et al. Identification of chronic rhinosinusitis phenotypes using cluster analysis. Int Forum Allergy Rhinol 2015;5:399-407.

41. Doulaptsi M, Prokopakis E, Seys S, et al. Visual analog scale for sino- nasal symptoms severity correlates with sino-nasal outcome test 22: paving the way for a simple outcome tool of CRS burden. Clin Transl Allergy 2018;8:32.

42. Bachert C, Zhang N, Hellings PW, Bousquet J. Endotype-driven care pathways in patients with chronic rhinosinusitis. J Allergy Clin Immunol 2018;141:1543-51.

43. Divekar R, Hagan J, Rank M, et al. Diagnostic utility of urinary LTE4 in asthma, allergic rhinitis, chronic rhinosinusitis, nasal polyps, and aspirin sensitivity. J Allergy Clin Immunol Pract 2016;4:665-70.

44. Baines KJ, Pavord ID, Gibson PG. The role of biomarkers in the management of airways disease. Int J Tuberc Lung Dis 2014; 18:1264-8.

45. Kim DW, Cho SH. Emerging endotypes of chronic rhinosinusitis and its application to precision medicine. Allergy Asthma Immunol Res 2017;9:299-306.

46. Benninger MS, Sindwani R, Holy CE, Hopkins C. Early versus delayed endoscopic sinus surgery in patients with chronic rhinosinusitis: impact on health care utilization. Otolaryngol Head Neck Surg 2015;152:546-52.

47. Hopkins C, Rimmer J, Lund VJ. Does time to endoscopic sinus surgery impact outcomes in chronic rhinosinusitis? Prospective findings from the National Comparative Audit of Surgery for Nasal Polyposis and Chronic Rhinosinusitis. Rhinology 2015;53:10-7.

48. Avdeeva K, Fokkens W. Precision medicine in chronic rhinosinusitis with nasal polyps. Curr Allergy Asthma Rep 2018;18:25.

49. Pinto JM, Mehta N, Di Tineo M, et al. A randomized, doubleblind, placebo-controlled trial of anti-IgE for chronic rhinosinusitis. Rhinology 2010;48:318-24.

50. Venera M, Picado C, Mullol J, et al. Efficacy of omalizumab in the treatment of nasal polyps. Thorax 2011;66:824-5.

51. Bidder, Sahota J, Rennie C, et al. Omalizumab treats chronic rhinosinusitis with nasal polyps and asthma together - a reallife study. Rhinology 2018;56:42-5.

52. Pillai P, Chan YC, Wu SY, et al. omalizumab reduces bronchial mucosa IgE and improves lung function in non-atopic asthma. Eur Respir J 2016;48:1593-601.

53. Ortega HG, Liu MC, Pavord ID, et al. Mepolizumab treatment in patients with severe eosinophilic asthma. N Engl J Med 2014;371:1198-207.

54. Gevaert P, Lang-Loidolt D, Lackner A, et al. Nasal IL-5 levels determine the response to anti-IL-5 treatment in patients with nasal polyps. J Allergy Clin Immunol 2006;118:1133-41.

55. Gevaert P, Van Bruaene N, Cattaert T, et al. Mepolizumab, a 
humanized anti-IL-5 mAb, as a treatment option for severe nasal polyposis. J Allergy Clin Immunol 2011;128:989-95.

56. Bachert C, Sousa AR, Lund VJ, et al. Reduced need for surgery in severe nasal polyposis with mepolizumab: a randomized trial. J Allergy Clin Immunol 2017;140:1024-31.

57. Weinstein SF, Katial RK, Bardin P, et al. Effects of Reslizumab on asthma outcomes in a subgroup of eosinophilic asthma patients with self-reported chronic rhinosinusitis with nasal polyps. J Allergy Clin Immunol Pract 2019;7:589-96.

58. Ghazi A. Benralizumab - a humanized MAb to IL-5Ra with enhanced antibody-dependent cell- mediated cytotoxicity - a novel approach for the treatment of asthma. Expert Opin Biol Ther 2011;12:113-8.

59. Tsurumaki H, Matsuyama T, Ezawa K, et al. Rapid effect of Benralizumab for hypereosinophilia in a case of severe asthma with eosinophilic chronic rhinosinusitis. Medicine (Kaunas) 2019;3:55

60. Kim H, Ellis AK, Fischer D, et al. Asthma biomarkers in the age of biologics. Allergy Asthma Clin Immunol 2017;13:48.

61. Bachert C, Manning L, Naclerio RM, et al. Effect of subcutaneous dupilumab on nasal polyp burden in patients with chronic sinusitis and nasal polyposis: a randomized clinical trial. JAMA 2016;315:469-79.

62. Jonstam K, Swanson BN, Mannent LP, et al. Dupilumab reduces local type 2 pro-inflammatory biomarkers in chronic rhinosinusitis with nasal polyposis. Allergy 2019;74:743-52.

63. Bachert C, Han JK, Desrosiers M, et al. Efficacy and safety of dupilumab in patients with severe chronic rhinosinusitis with nasal polyps (LIBERTY NP SINUS-24 and LIBERTY NP SINUS-52): results from two multicenter, randomized, doubleblind, placebo-controlled, parallel-group phase 3 trials. Lancet 2019;394:1638-50.

64. Kartush AG, Schumacher JK, Shah R, Patadia MO. Biologic agents for the treatment of chronic rhinosinusitis with nasal polyps. Am J Rhinol Allergy 2019;33:203-11.

65. Ciprandi G, Tosca MA, Silvestri M, Ricciardolo FLM. Inflammatory biomarkers for asthma endotyping and consequent personalized therapy. Expert Review Clin Immunol 2017; 13:715-21.

66. Ellis AK, Tenn MW. Advances in rhinitis: Models and mechanisms. Ann Allergy Asthma Immunol 2018;121:61-64

67. Ciprandi G, Varricchio A, Tajana G, et al. Peripheral eosinophils correlate with nasal eosinophils in patients with rhinitis. J Invest Allergol Clin Immunol 2018;28:428-30.

68. Ciprandi G, Silvestri M. Standardization of the nasal cytology in the workup of allergic rhinitis. Ann All Asthma Immunol 2019;123:213-6.

69. Ricciardolo F, Sorbello V, Ciprandi G. FeNO as a biomarker for asthma phenotyping and management. Allergy Asthma Proc 2015;36:1-8.

70. Gelardi M, Abbatista G, Quaranta VN, et al. Standardization procedure for the nasal nitric oxide measurement method using Niox MINO ${ }^{\circledR}$ and the tidal- breathing technique with velumclosure. J Biol Res 2016;30:853-858.

71. Ricciardolo FLM, Levra S, Sprio AE, et al. A real-world assessment of asthma with chronic rhinosinusitis. Ann Allergy Asthma Immunol 2020;125:65-71.

72. Roth-Walter F, Adcock IM, Benito-Villalvilla $\mathrm{C}$, et al. Comparing biologicals and small molecule drug therapies for chronic respiratory diseases: an EACCI Taskforce on the immunopharmacology position paper. Allergy 2019;74:432-48.

73. Gelardi M, Fiorella ML, Russo C, Fiorella R, Ciprandi G. Role of nasal cytology. Int J Immunopathol Pharm 2010;23:45-9.

74. Gelardi M, Iannuzzi L, De Giosa M, et al. Non-surgical management of chronic rhinosinusitis with nasal polyps based on clinical cytological grading: a precision medicine-based approach. Acta ORL Ital 2017;37:38-45.

75. Gelardi M, Piccininni K, Quaranta N, et al. Olfactory dysfunction in patients with chronic rhinosinusitis with nasal polyps is associated with clinical-cytological grading severity. Acta Otorhinolaryngol Ital 2019;39:329-35.

76. Gelardi M, Porro G, Quaranta V, et al. Clinical-cytologicalgrading and phenotyping in patients with chronic rhinosinusitis with nasal polyps: the relevance in clinical practice. Monaldi Arch Chest Dis 2020;90:1277.

77. Gelardi M, Cassano M, Ciprandi G. The clinical relevance of the clinical cytological grading in patients with chronic rhinosinusitis with nasal polyps. J Allergy Clin Immunol 2020;146:462-3.

78. Soler ZM, Jones R, Le P, et al. SNOT-22 outcomes after sinus surgery: a systematic review and meta-analysis. Laryngoscope 2018;128:581-92.

79. Klimek L, Spielhaupter M, Alali A, et al. Elevated eosinophil count associated with dupilumab therapy in patients with type2 inflammation. Laryngorhinootologie 2020;99:811-2.

80. Bachert C, Zhang N, Cavaliere C, et al. Biologics for chronic rhinosinusitis with nasal polyps. J Allergy Clin Immunol 2020;145:725-39.

81. Tsetsos N, Markou K, Konstantidinis I. Effect of monoclonal antibodies on olfactory dysfunction caused by chronic rhinosinusitis with nasal polyps: a systematic review and meta-analysis. Int Forum Allergy Rhinol 2020;10:893-900.

82. Licari A, Castagnoli R, De Filippo M, et al. Current and emerging biologic therapies for allergic rhinitis and chronic rhinosinusitis. Exp Opin Biol Ther 2020;20:609-9. 